\title{
Eventmarketing - Prosumtionstheoretische Implikationen zur Erweiterung der Theoriebildung eines innovativen Kommunikationsinstruments
}

\author{
Frank Sistenich, Katrin Böckler
}

\section{Zusammenfassung}

Klassische Massenkommunikation erreicht relevante Zielgruppen bei fortschreitender Werbedichte und wachsenden Reaktanzreaktionen von Konsumenten nicht mehr hinreichend. Unternehmen setzten daher heute auf innovative Kommunikationskonzepte, um Marketingbotschaften zu senden. Neue Konzepte wie das Open SourceMarketing integrieren den Konsumenten aktiv in Vermarktungs- und Absatzprozesse und können Botschaften authentischer und glaubwürdiger transportieren. Konsumenten beteiligen sich somit aktiv an den Wertschöpfungsprozessen von Unternehmen. In der Literatur wird diese Integration als »Prosumtion« bezeichnet, die verschiedene Ausprägungen kennt. Der vorliegende Beitrag zeigt auf, welche Rolle der Prosument im Eventmarketing einnimmt. Eventmarketing als modernes Kommunikationsinstrument zeichnet sich durch seine Dialog- und Interaktionsorientierung aus. Während eines Marketingevents werden Konsumenten aktiv über die konative Verhaltensebene in die Eventinszenierung einbezogen. Die Ansätze der Prosumentenforschung eignen sich daher, die Eventforschung theoretisch sinnvoll zu ergänzen und zu erweitern.
Abstract

Traditional mass communication is no longer sufficient to reach customer target groups. Therefore modern instruments of marketing communication have been developed in recent years. One prominent example is the case of event marketing. Due to its characteristics of interaction the theoretical framework can be extended by the approach of Tofflers prosumtion theory. Prosumtion is a leading trend since the 80ies and well recognized in marketing theory in the field of product-, price and distribution policy. For the framework of event marketing theory prosumtion is an especially valuable asset to enhance important theory building.

\section{1 »Der Aufstieg des Prosumenten« nach Alvin Toffler (1980)}

Der amerikanischer Soziologe und Futurologe Alvin Toffler beschreibt 1980 in seinem Buch »The Third Wave« den Wandel der Gesellschaft (Toffler 1980: 16). Toffler prognostiziert eine neue, hoffnungsvolle Epoche der Menschheit, in der die bestehende Industriegesellschaft mit ihren Wertvorstellungen in Bezug auf Familie, Kirche und Staat von einer neuen Zivilisation abgelöst wird. Der Autor teilt die Menschheitsgeschichte zur Entwicklung und Beschreibung des neuen Zivilisationsstatus in drei Epochen bzw. sogenannte Veränderungswellen ein, die jeweils variierende Tätig- keitsprofile von Produzent und Konsument aufweisen und im Fokus seiner Betrachtung liegen.

Die erste Welle beginnt bei Toffler mit dem Entstehen der Landwirtschaft, ausgelöst durch die Agrarrevolution ca. 8.000 v. Chr. (Toffler 1980: 24-25). Während dieser Epoche »konsumierten die Menschen, was sie selbst produzierten « (Toffler 1980: 273). Toffler spricht hier von »Produktion für den Eigenverbrauch « (Toffler 1980: 273), also einer Produktion, die zum Zweck der Selbstversorgung betrieben wird. Konsument und Produzent kamen demnach in Personalunion (Hellmann 2010: 16) und als geschlossenes Ganzes vor (Toffler 1980: 49). Es handelte sich folglich um eine »Verzahnung der beiden Tätigkeitsprofile Produzent (...) und 
Konsument (...) [, die] den Neologismus Prosument « bilden (Hellmann 2010: 17).

Im Zuge der Industriellen Revolution gegen Ende des 17. Jahrhunderts folgte eine zweite, industriell geprägte Welle (Toffler 1980: 25, 273). Diese zweite Innovationswelle schuf eine gespaltene Wirtschaftsform, die für die Trennung von Produktion und Konsumtion sowie für eine damit einhergehende Ausbreitung der Märkte steht (Toffler 1980: 51). Die Produktion diente nun kaum noch dem Eigenverbrauch, sondern vornehmlich der Befriedigung der Nachfrage auf einem Markt bzw. der industriellen »Produktion für den Markt«. Im Rahmen dieser Entwicklung teilt Toffler die Wirtschaft in zwei Sektoren ein.

Sektor A - der Prosumtionssektor - umfasst die unbezahlte Arbeit und somit den Bereich der »Produktion für den Eigenverbrauch «. Sektor B - der Produktionssektor - beinhaltet die bezahlte Arbeit, also die Produktion von Gütern und Dienstleistungen für den Markt. Als produktiv bzw. ökonomisch relevant galt nur die Arbeit in Sektor B, womit der Prosument vorerst in Vergessenheit geriet (Toffler 1980: 273).

Toffler betrachtet diese Entwicklung der damaligen Gesellschaftsordnung kritisch und geht z. B. davon aus, dass dadurch die Ausweitung des Produktionssektors und eine damit verbundene Expansion der Marktwirtschaft, eine aggressive Erwerbsethik, die mit Erfolgsdruck einhergeht und sich negativ auf das Leben der Menschen auswirkt, zu verzeichnen ist. Die Lösung für dieses Problem sieht der Autor in einem ausgeglichenen Verhältnis beider Sektoren, welches in der folgenden Innovationswelle zustande kommen soll (Toffler 1980: 386-387).

Während der dritten Welle - dem Zeitalter des Prosumenten - rückt der Prosument wieder ins Zentrum des wirtschaftlichen Lebens (Toffler 1980: 281). Toffler sieht Anzeichen für diesen Umbruch während der 1970er Jahre. Zu diesem Zeitpunkt stellt z. B. die Pharmaindustrie Schwangerschaftstests zur Selbstuntersuchung zur Verfügung (Toffler 1980: 272). Als weitere Indikatoren für eine neue Veränderungswelle benennt er verschiedene Beispiele, die zeigen, dass immer mehr Tätigkeiten aus dem Sektor B - der bezahlten Arbeit - in den Sektor A abwandern (Toffler 1980: 272, 276-281). Folglich werden Güter und Dienstleistungen wieder für den Eigenverbrauch produziert. Mit dem Aufstieg des Prosumenten wird es zu einer Auflösung des Marktes kommen (Toffler 1980: 289). Die daraufhin neu entstehende Zivilisation wird sich »lebhafter voneinander unterscheiden [...] und ein höheres Maß an Individualität an den Tag legen« (Toffler 1980: 390). Es wird ein ausgeglichenes Verhältnis von Arbeit und Freizeit sowie ein menschliches Miteinander im Mittelpunkt stehen (Toffler 1980: 390).

\section{Soziologie des Prosumenten - Ein Tätigkeitsprofil nach Hellmann (2010)}

Toffler geht davon aus, dass sich die Tätigkeitsprofile von Konsumenten und Produzenten zunehmend annähern, vermischen und wechselseitig ergänzen (Hellmann 2010: 26). Ungeklärt bleibt bei Toffler jedoch, wo Konsumtion aufhört und wo Prosumtion beginnt (Hellmann 2010: 25). Zur Abgrenzung des Prosumentenbegriffs untersucht Hellmann (2010: 26) daher die Tätigkeitsprofile des Konsumenten und Produzenten näher, um darauf aufbauend das Profil des Prosumenten systematisch herzuleiten.

Ausgangspunkt für Hellmanns Herangehensweise ist die Annahme, dass sich Konsumenten in der Publikumsrolle und Produzenten in der Leistungsrolle des Wirtschaftssystems befinden (Hellmann 2010: 26).

Publikumsrollen sind primär auf einen Leistungsempfang ausgerichtet. Konsumenten befinden sich in der Publikumsrolle des Wirtschaftssystems und zeichnen sich demzufolge durch ein vorwiegend passives Verhalten aus. Dazu scheint ein aktiver Konsument, wie Toffler ihn beschrieben hat, widersprüchlich zu sein. Jedoch hat die Forschung gezeigt, dass Konsumenten nicht generell passiv agieren, beispielsweise zeigt sich bei der Rezeption von Massenmedien, dass ein gewisses Maß an Aktivität vorhanden sein muss, da reine Passivität eine Nullrezeption zur Folge hätte (Hellmann 2010: 27; Stauff 2004: 67).

Leistungsrollen sind primär auf eine Leistungserbringung ausgerichtet. Produzenten befinden sich in der Leistungsrolle des Wirtschaftssystems und zeichnen sich demzufolge durch ein aktives Verhalten und den Faktor Arbeit aus (Hellmann 2010: 30). Daher stellt Hellmann verschiedene Definitionen von Arbeit vor, um herauszustellen, wann jemand arbeitet, und er resümiert: »Hat etwas Gebrauchswert für jemanden, [...] handelt es sich um eine Leistung, und wurde diese Leistung von jemandem extra hergestellt, hat diese Person dafür gearbeitet« (Hellmann 2010: 33). Demnach wird »immer dort gearbeitet, wo [...] am Ende eines Herstellungsprozesses eine bestimmte Sach- oder Dienstleis- 
tung für irgendjemanden Gebrauchs- und ggf. auch Tauschwert besitzt « (Hellmann 2010: 35).

Hellmann leitet aus Tofflers Ausführungen und seiner systematischen Verortung des Prosumenten sowie dessen Tätigkeitsprofil folgende Definition ab: »Prosumtion liegt immer dann vor, wenn zur Herstellung einer Sach- oder Dienstleistung, die vor allem für die Eigenverwendung gedacht ist und von daher ihren Gebrauchswert bezieht, ein Beitrag geleistet wird, ohne den der Herstellungsprozess unabgeschlossen bleibt, unabhängig davon, ob für diese Leistung bezahlt werden muss oder nicht« (Hellmann 2010: 36).

Prosumtion lässt sich folglich nur dadurch vom aktiven Konsum abgrenzen, dass »der Herstellungsprozess bei Entzug der Prosumtionsaktivitäten zu keinem Abschluss käme, während der Herstellungsprozess im Falle des Entzugs einer Konsumtionsaktivität davon unberührt bliebe« (Hellmann 2010: 36). Demnach kann nicht von Produktion, sondern es muss von Prosumtion gesprochen werden, wenn jemand größtenteils für sich selbst arbeitet und dabei Gebrauchswert schafft.

\section{Implikationen aus Hellmanns Ansatz für die moderne Eventforschung}

Die Ausführungen Hellmanns können auch anhand der Tätigkeitsprofile von Eventteilnehmern und -veranstaltern nachvollzogen werden. Zwar handelt es sich beim Eventmarketing um ein Kommunikationsinstrument (Zanger/Sistenich 1996: 233ff; auch Bruhn 2010: 204), dennoch scheint es sinnvoll, die erlebnisorientierte Vermittlung von Marketingbotschaften in diesem Rollenverhältnis zu betrachten. Dies begründet sich einerseits in der Tatsache, dass in der Literatur besonders der soziologische Ansatz der Rollentheorie für eine theoretische Fundierung des Eventmarketings fruchtbar scheint (Sistenich 1999). Andererseits kommen auch während eines Marketingevents Publikums- und Leistungsrollen zum Tragen.

\subsection{Tätigkeitsprofil des Eventteilnehmers}

Publikumsrollen sind primär auf einen Leistungsempfang ausgerichtet und zeichnen sich durch ein vorwiegend passives Verhalten aus (Hellmann 2010: 27). Eventteilnehmer befinden sich während eines Events in einer Publikumsrolle. Bezogen auf das Eventmarketing zeigt sich jedoch anhand der Charakteristika von Marketingevents, dass die Rolle der Eventteilnehmer nicht generell passiv ausgelegt ist. Eventteilnehmer werden ferner über die Verhaltensebene in die Eventinszenierung einbezogen (Zanger/Sistenich 1996: 235) und beteiligen sich dementsprechend aktiv an dem Event. Die Teilnehmer treten folglich aus ihrer klassischen Publikumsrolle, die durch passives Zuschauen geprägt ist, heraus.

Restriktiv kann in diesem Zusammenhang festgehalten werden, dass sich Eventteilnehmer auch passiv verhalten können und sich lediglich auf das Zuschauen beschränken. Dies verdeutlicht z. B. die Adidas Streetball Challenge. Einerseits beteiligten sich Eventteilnehmer aktiv über die konative Verhaltensebene an dem Marketingevent, indem sie während des Events Basketball spielten (Eventteilnehmer als Spieler) und so in die erlebbare Markenwelt eintauchten. Andererseits wurden sie dabei von einem teilnehmenden Publikum begleitet, dass sich lediglich auf das Zuschauen beim Event beschränkte (Eventteilnehmer als Zuschauer). Spieler und Zuschauer können als Eventteilnehmer betrachtet werden. Dies verdeutlicht, dass auch beim Event, ähnlich wie bei der von Hellmann beschriebenen Konsumtion, die Teilnahme passiv sowie aktiv sein kann.

\subsection{Tätigkeitsprofil des Eventveranstalters}

Leistungsrollen sind primär auf eine Leistungserbringung ausgerichtet (Hellmann 2010: 30). Eventveranstalter befinden sich während des Events in der Leistungsrolle und zeichnen sich demzufolge durch ein aktives Verhalten aus. Dem Eventveranstalter obliegt der Eventmarketingprozess, welcher aus der strategischen und operativen Vorbereitung, der Durchführung sowie der Nachbereitung besteht (Zanger 2010: 6). Während des eigentlichen Marketingevents übernimmt der Eventveranstalter z. B. die Begrüßung der Teilnehmer, setzt die Inszenierung der Ereignisse um oder koordiniert das Interaktionsangebot an die Eventteilnehmer. Außerdem werden »dem Adressaten (Kunden, Händler, Meinungsführer, Mitarbeiter) [im Rahmen des Marketingevents] firmen- oder produktbezogene Kommunikationsinhalte erlebnisorientiert vermittel[t] « (Zanger 2010: 5). Der Eventveranstalter kann bei den einzelnen Prozessschritten auch auf Dienstleister zurückgreifen und z. B. eine Eventagentur zur Vorbereitung beauftragen. Im Falle der Adidas Streetball Challenge trat Adidas dominant als Veranstalter auf (Nufer 2007: 108) und übernahm bei der »Durchführung [...] die Projektleitung, die Kommunikation und die Personalausstat- 
tung « (Nufer 2007: 109). Aufgaben aus dem Bereich der Logistik wurden von externen Partnern übernommen (Nufer 2007: 109).

\subsection{Tätigkeitsprofil des Event-Prosumenten}

Aufbauend auf den Tätigkeitsprofilen von Konsumenten und Produzenten entwickelt Hellmann eine Definition des Prosumenten. Aus seinen Ausführungen kann eine Definition von Prosumtion im Eventmarketing hergeleitet werden. Betrachtet man auf Basis von Hellmanns Begriffsabgrenzung der Prosumtion den Eventteilnehmer als Prosumenten und überprüft dessen Tätigkeitsprofil, so betätigen sich Eventteilnehmer als Event-Prosumenten, wenn sie sich aktiv über die Verhaltensebene an einem Marketingevent beteiligen und das Interaktionsangebot nutzen. Folglich wird der passive Zuschauer eines Events nicht als Event-Prosument bezeichnet.

Eine weitere Möglichkeit zur Prosumtion im Eventmarketing stellt die aktive Beteiligung des Eventteilnehmers am Eventmarketingprozess dar. Hier ist beispielsweise vorstellbar, dass sich Teilnehmer an der operativen Planung von Events beteiligen, indem sie an der Konzeption der Eventideen mitwirken. Denkbar wäre z. B., dass Fans des Lifestyle-Getränks Red Bull Anregungen für neue Events liefern, die die Botschaft »Red Bull verleiht Flügel « erlebbar machen. Im Rahmen der operativen Umsetzung ist denkbar, dass Event-Prosumenten an der Vorfeldkommunikation des Events mitwirken und ihr Umfeld über interessante Events informieren.

Event-Prosumenten können sich weiterhin an der Durchführung des Events beteiligen. Auf dieser Ebene wird die Eventkonzeption umgesetzt. Dies beinhaltet z. B. alle geplanten Aktionen des Events, wie »Moderatorenauftritte, Showacts, Auszeichnung von Preisträgern« usw. (Zanger/Drengner 2009: 206). Eventteilnehmer können als Prosumenten auftreten, indem z. B. während des Red Bull Cliff Diving-Events der Sieger des letzten Contests den neuen Sieger auszeichnet. In diesem Fall wird die Siegerehrung folglich nicht vom Eventveranstalter durchgeführt, sondern obliegt einem Eventteilnehmer. Allerdings handelt es sich nur um Prosumtion im Eventmarketingprozess, wenn die Prosumtionsaktivität vor allem für die Eigenverwendung gedacht ist. Für die Event-Prosumenten des Red Bull Cliff Diving-Events, bedeutet dies, dass vor allem die Darstellung des eigenen Lifestyles als Motiv angesehen werden kann.
Abschließend können Eventteilnehmer sich auch an der Nachbereitung von Events beteiligen. Im Nachfeld werden z. B. »Videos und Bilder der Veranstaltung durch Außendienstmitarbeiter an die Eventteilnehmer « übergeben (Zanger/Drengner 2009: 207). An dieser Nachbereitung können Event-Prosumenten mitwirken, indem sie eigene Impressionen des Events in Internetportalen wie YouTube darstellen oder auch in sozialen Netzwerken wie Facebook über das Marketingevent berichten. Die Red Bull-Events können auch direkt auf der Webseite kommentiert werden (Red Bull GmbH 2011). Durch diese Möglichkeit wird Event-Prosumtion unmittelbar vom Unternehmen bzw. von Red Bull angeregt. Der Eventteilnehmer kann auch als Multiplikator der Eventbotschaft gesehen werden. Teilnehmer von Events beteiligen sich damit am Marketing bzw. an der Kommunikationspolitik von Unternehmen, indem durch Word of Mouth-Aktivitäten Botschaften weitergetragen werden (Hellmann 2010: 37). Jedoch sollen Botschaften und auch die Markenwelt während eines Events unmittelbar erlebt werden und können daher kaum adäquat über Word of Mouth-Aktivitäten transportiert werden. Daher steht dies nicht im direkten Zusammenhang mit Prosumtion im Eventmarketing, sondern eher mit Prosumtion im Marketing an sich.

Die folgende Abbildung 1 fasst die aufgezeigte Prosumtion im Eventmarketing zusammen.

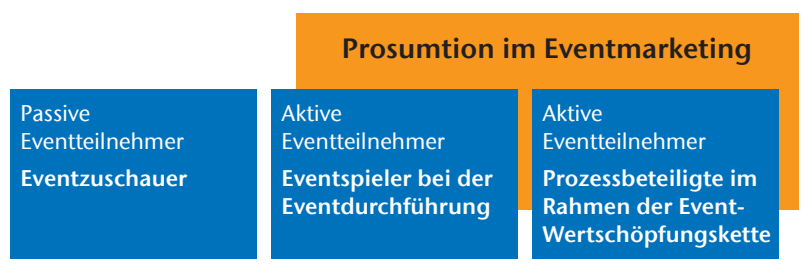

Abb. 1: Prosumtion im Eventmarketing (Quelle: Eigene Darstellung)

In Anlehnung an Hellmann wird folgende neue begriffliche Abgrenzung des Prosumenten im Eventmarketing vorgeschlagen: Eventteilnehmer betätigen sich immer dann als Prosumenten, wenn ohne deren aktives Handeln auf einer konativen Ebene der Charakter eines Events nicht zum Tragen kommen würde (s. Charakteristika von Marketingevents in: Zanger 2010: 6-7; Zanger/Sistenich 1996: 233-234) und somit Eventziele auch nicht realisiert werden können. Dabei handelt es sich im Regelfall um kollektive Prosumtion, d. h. dass es nicht unbedingt auf jeden einzelnen Eventteilnehmer ankommt, jedoch die kollektive Masse entscheidend ist, um das Eventziel zu erreichen und die Eventbotschaft zu verbreiten. 


\section{Die Funktion inszenierter Prosumtion nach Panzer (2010)}

Gerhard Panzer erläutert 2010 in seinem Beitrag »Die Funktion inszenierter Prosumtion für Qualität und Wert kultureller Güter« die Bedeutung des Prosumenten im Kunst- und Kulturmarkt und analysiert mögliche Arten von Prosumtion für verschiedene Werkformen (Panzer 2010: 132).

Zur Abgrenzung von Kulturkonsumenten und Kulturprosumenten beschreibt Panzer zuerst die Konsumenten, welche Kultur vorwiegend konsumieren, während sie kulturelle Werke wahrnehmen, erwerben und nutzen (Panzer 2010: 138). Kulturkonsum unterscheidet sich vom übrigen Konsum durch die Tatsache, dass »in der Regel keine Güter verbraucht und andere Nutzer nicht zwangsläufig ausgeschlossen werden« (Panzer 2010: 138).

Konsumenten werden in diesem Markt zu Prosumenten, indem sie durch ihre Beiträge den Sinngehalt künstlerischer Werke anreichern und erweitern (Panzer 2010: 137). Jedoch stellt sich hier die Frage, ob nicht bei Kunstrezeption, Kunstgenuss und Kunstwahrnehmung ebenfalls von Prosumtion gesprochen werden kann, da der Sinn und Zweck von Kunst nur erfüllt wird, wenn der Kulturkonsument ihr genügend Aufmerksamkeit schenkt. Zentrales Merkmal der Prosumtion im Kunstund Kulturmarkt ist eine dialogische Struktur zwischen Konsumenten und Produzenten, d. h. dass für sie Partizipation und Kooperation im Vordergrund stehen (Panzer 2010: 137).

Im Bereich der Kunst und Kultur finden sich verschiedene Arten von Prosumtion. Um diese Arten herauszufiltern, unterscheidet Panzer in einem ersten Schritt zwischen einer festen und einer offenen künstlerischen Werkform (Panzer 2010: 139). Feste Werkformen wie z. B. Kunstwerke in der Bildenden Kunst und offenen Werkformen, wie sie im Bereich der darstellenden Künste z. B. als Theater zu finden sind, »differieren dadurch, ob ein fixiertes Werk konsumiert wird, oder ob es während des Konsums in Ko-Präsenz [...] entsteht « (Panzer 2010: 139).

Bei festen künstlerischen Werkformen findet Prosumtion nachträglich statt und ergänzt das Werk. Panzer spricht in diesem Fall von rahmender sekundärer Prosumtion, die additiv stattfindet und »keinen primären Einfluss auf die inhaltliche Qualität des Werkes hat « (Panzer 2010: 140).
Bei offenen künstlerischen Werkformen findet Prosumtion in den verschiedenen Stufen der Werkproduktion statt und ist daher vielfältiger. Die erste Stufe beinhaltet die Werkschöpfung, in der z. B. Autoren von Theaterstücken ein Werk schaffen. Hier findet keine Kooperation und keine Prosumtion statt. Auf einer zweiten Stufe wird z. B. von Regisseuren eine Inszenierung in Zusammenarbeit mit Schauspielern erarbeitet, wobei die Beteiligten interaktiv kooperieren. Während der dritten Stufe, der Aufführung, üben Zuschauer Einfluss auf die Aufführung aus. Auf dieser Stufe werden somit Konsumenten zu Prosumenten (Panzer 2010: 140).

Laut Panzer bieten diese offenen Werkformen diverse Möglichkeiten zur Prosumtion. Jedoch kann bei der zweiten Stufe, der Inszenierung, nicht von Prosumtion gesprochen werden, da Regisseur und Schauspieler das Ursprungswerk beeinflussen und folglich als Produzenten betrachtet werden müssen. Dennoch wird aus Panzers Ausführungen deutlich, dass Prosumenten im Kunst- und Kulturmarkt in verschiedenen Formen aktiv werden. Kulturprosumtion kann in diesem Bereich folglich nicht nur als Phänomen, sondern als konstitutiver Beitrag zu künstlerischen Werken betrachtet werden (Panzer 2010: 141).

Die gewonnenen Erkenntnisse aus der Prosumentenforschung in Bezug auf Kunst und Kultur werden vor allem von der Theaterwissenschaft genutzt, um auf das Publikum zuzugreifen und dies während einer Inszenierung gezielt zu steuern. Die Zuschauer werden aus ihrer passiven Rolle herausgeholt und zur interaktiven Beeinflussung einer Aufführung angeregt. Das macht sie zu einem konstitutiven Teil der Aufführung und zu einem Kulturprosumenten. Mit Hilfe dieser Einbeziehung des Publikums können künstlerische Inhalte authentischer erschlossen werden (Panzer 2010: 141-142). Prosumtion findet auch in anderen kulturellen Sparten außerhalb der Theaterwissenschaft statt. Daraus folgert Panzer, dass Prosumtion heute gezielt inszeniert wird und daher von »inszenierter Prosumtion « gesprochen werden kann. Zur näheren Betrachtung dieser Prosumtion zieht der Autor den Begriff der »Inszenierung « in der Theaterwissenschaft heran (Panzer 2010: 142). Diese definieren die »Inszenierung als den Vorgang der Planung, Erprobung und Festlegung von Strategien « (Fischer-Lichte 2004: 327). Diese Definition wird dahingehend erweitert, dass zusätzlich »eine Situation geschaffen wird, die Frei- und Spielräume für nicht-geplante, nicht inszenierte Handlungen, Verhaltensweisen und Ereignisse eröffnet« (Fischer-Lichte 2004: 327). 
Inszenierte Prosumtion heißt, dass die Prosumtion "zum einen planend und systematisch herbeigeführt [wird], zum anderen für eine künstlerische Beteiligung der Konsumenten erweitert [wird]. Es wird zielgerichtet ermöglicht, die inhaltliche Qualität von `Werken $`$ nicht nur zu beeinflussen, sondern auch deren Aussage mit zu prägen« (Panzer 2010: 144).

Toffler prognostizierte einen eigenständigen Aufstieg des Prosumenten (Toffler 1980). Heute zeigt sich jedoch, dass die Prosumtion gezielt ausgelöst und inszeniert werden muss, um sich im Rahmen des Kunst- und Kulturmarktes zu entwickeln (Panzer 2010: 144).

\section{Implikationen aus Panzers Ansatz für die moderne Eventforschung}

Panzer erläutert in seinem Ansatz die Bedeutung von inszenierter Prosumtion für den Kunst- und Kulturmarkt (Panzer 2010) und analysiert die Prosumtion für den Kulturkonsum, also für ein Konsumgut. Beim Eventmarketing kann nicht vom Konsumieren eines Events gesprochen werden, da Events kein Konsumgut darstellen, sondern ein Kommunikationsinstrument sind. Im Vordergrund eines Marketingevents steht daher die Inszenierung einer Markenwelt und nicht ein inszeniertes Kunst- und Kulturprodukt, auch wenn dieses im Einzelfall ein Kulturgut sein kann. Dennoch können Panzers Ausführungen herangezogen werden, um eine mögliche inszenierte Prosumtion im Eventmarketing näher zu untersuchen.

Panzer zeigt auf, dass im Kunst- und Kulturmarkt Prosumtion anzufinden ist, die sich anhand verschiedener Werkformen nachweisen lässt. Allerdings vollzieht sich die Prosumtion in diesem Markt, entgegen Tofflers Auffassung vom »Aufstieg des Prosumenten«, nicht eigenständig, d. h. dass Konsumenten nicht nur »von sich aus « zu Prosumenten werden und sich an der Produktion beteiligen (Panzer 2010: 144). Heute wird anhand des Kunst- und Kulturmarktes deutlich, dass Prosumtion auch im Rahmen einer strategischen Perspektive extra herbeigeführt, beeinflusst und gesteuert werden kann, indem jene inszeniert wird. Dies erschließt neue Möglichkeiten zur Betrachtung von Prosumtion im Eventmarketing und erweitert dessen theoretische Betrachtung.

In den vorangegangenen Ausführungen zum Prosumenten im Eventmarketing wurde dargelegt, dass unter bestimmten Bedingungen sehr wohl von Event-Pro- sumtion gesprochen werden kann. Wie oben erläutert, betätigen sich Eventteilnehmer dann als Event-Prosumenten, wenn sie sich aktiv über die Verhaltensebene an einem Marketingevent beteiligen und das Interaktionsangebot nutzen. Kann jedoch beim Eventmarketing von inszenierter Prosumtion gesprochen werden? Events als inszenierte Ereignisse in Form von Veranstaltungen und Aktionen (Zanger 2010: 5) beinhalten Elemente einer Inszenierung. Daher scheint es schlüssig, Panzers Ansatz heranzuziehen, um zu überprüfen, ob es sich bei jener Prosumtion im Eventmarketing um inszenierte Prosumtion handelt.

Zur näheren Untersuchung der inszenierten Prosumtion wird die oben genannte Definition Panzers herangezogen. Aus der Verbindung dieser Definition mit der neu entwickelten Definition von Event-Prosumtion wird folgende neue begriffliche Abgrenzung von inszenierter Prosumtion im Eventmarketing vorgeschlagen:

»Inszenierte Event-Prosumtion liegt vor, wenn ein aktives Handeln des Eventteilnehmers auf einer konativen Ebene und ein entsprechendes Interaktionsangebot einerseits planend und systematisch herbeigeführt wird und andererseits während des Events Frei- und Spielräume für nicht geplante, nicht inszenierte Handlungen, Verhaltensweisen und Ereignisse geschaffen werden.«

Im Eventmarketing wird vorab geplant, wie sich Eventteilnehmer während eines Events aktiv über die Verhaltensebene einbringen können und sollen. Es wird also im Zuge der operativen Vorbereitung eines Events überlegt, wie das Interaktionsangebot, das zum Erleben der Markenwelt beiträgt, aussehen soll. Die Ereignissituation eines Events wird bewusst vom Eventveranstalter organisiert. Beteiligen sich nun die Event-Prosumenten an der vorab durchdachten Interaktionssituation und nutzen sie Frei- und Spielräume, um sich individuell einzubringen, kann von inszenierter Prosumtion im Eventmarketing gesprochen werden.

Mithilfe des vorab geplanten und während des Events geleiteten Interaktionsangebots können im Rahmen eines Marketingevents Erlebnisse geschaffen werden, die sich von der Alltagswirklichkeit der Eventteilnehmer unterscheiden. Die eröffneten Frei- und Spielräume während eines Marketingevents ermöglichen eine authentische Gestaltung der Interaktionssituationen und somit auch eine glaubwürdige Vermittlung der Markenwelt. Das inszenierte Ereignis kann zu einer nachhaltigen Beeinflussung der Konsumenteneinstellung beitragen. 


\section{Fazit}

Tofflers These vom »Aufstieg des Prosumenten« hat bis heute seine Gültigkeit behalten. Konsumenten beteiligen sich gegenwärtig zunehmend auf verschiedene Art und Weise aktiv handelnd an den Wertschöpfungsprozessen von Unternehmen und werden somit zu Prosumenten. Dies gilt für alle Instrumente des MarketingMixes. Eine potentiell besondere Bedeutung hat der Ansatz für die Eventforschung, da sich das Eventmarketing durch einen hohen konativen Handlungsbezug des Konsumenten von anderen kommunikationspolitischen Instrumenten unterscheidet. Interessant für den Bezug des Prosumenten im Eventmarketing sind die Ansätze von Hellmann und Panzer. Diese können herangezogen werden, um die Eventforschung theoretisch weiterzuentwickeln und somit die Bedeutung der Prosumtion im Eventmarketing darzulegen.

Eventteilnehmer betätigen sich dann als EventProsumenten, wenn sie sich aktiv über die Verhaltensebene an einem Marketingevent beteiligen und das Interaktionsangebot im Rahmen einer konativen Aktivierung nutzen. Folglich kann der passive Zuschauer eines Events nicht als Event-Prosument bezeichnet werden. Eine weitere Möglichkeit zur Abgrenzung des Event-Prosumenten ergab sich durch die Analyse des Eventmarketingprozesses. Prosumenten können sich auf verschiedenen Ebenen am Wertschöpfungsprozess beteiligen, sofern Interaktionsangebote geschaffen werden. Aus diesen Schlussfolgerungen wurde eine Definition zur Abgrenzung des Prosumenten im Eventmarketing aufgestellt. Es konnte aufgezeigt werden, dass Eventteilnehmer während eines Events in vielerlei Hinsicht als Event-Prosumenten agieren können und sich aktiv an Marketingevents und an dessen vor- und nachgelagerten Prozessen beteiligen.

In der Weiterführung von Panzers Ausführungen für eine inszenierte Prosumtion im Eventmarketing wurde deutlich, dass Frei- und Spielräume während eines Marketingevents eine authentische Gestaltung der Interaktionssituationen sowie eine glaubwürdige Vermittlung der Markenwelt ermöglichen.

Diese Überlegungen bieten eine Grundlage, um die Prosumtion im Eventmarketing näher zu untersuchen und einen fruchtbaren Ansatz, die theoretische Basis dieses innovativen Kommunikationsinstruments weiterzuentwickeln.

\section{Literatur}

Bruhn, M. (2010): Marketing: Grundlagen für Studium und Praxis. Gabler, Wiesbaden.

Fischer-Lichte, E. (2004): Ästhetik des Performativen. Suhrkamp, Frankfurt am Main.

Hellmann, K.-U. (2010): Prosumer Revisited: Zur Aktualität einer Debatte. Eine Einführung, in: Blättel-Mink, B., Hellmann, K.-U. (Hg.): Prosumer revisited: Zur Aktualität einer Debatte, Verlag für Sozialwissenschaften, Wiesbaden, 13-48.

Nufer, G. (2007): Event-Marketing und -Management: Theorie und Praxis unter besonderer Berücksichtigung von Imagewirkungen. Deutscher Universitätsverlag, Wiesbaden.

Panzer, G. (2010): Die Funktion inszenierter Prosumtion für Qualität und Wert kultureller Güter. In: Blättel-Mink, B., Hellmann, K.-U. (Hg.): Prosumer revisited: Zur Aktualität einer Debatte, Verlag für Sozialwissenschaften, Wiesbaden, 131-145.

Red Bull GmbH (2011): Red Bull Cliff Diving Videos: Am Tempel des Poseidon, http://www.redbull.de/cs/Satellite/de_DE/Video/AmTempel-des-Poseidon-021242782042971, Zugriff: 17.08.2011.

Sistenich, F. (1999): Eventmarketing. DUV Verlag, Wiesbaden.

Stauff, M. (2004): Der Konsum der Zuschauer: Zur televisuellen Umwertung von Wahrnehmungen und Bedeutungen, in: Hellmann, K.-U., Schrage, D. (Hg.): Konsum der Werbung: Zur Produktion und Rezeption von Sinn in der kommerziellen Kultur, Verlag für Sozialwissenschaften, Wiesbaden, 63-80.

Toffler, A. (1980): Die Zukunftschance: Von der Industriegesellschaft zu einer humaneren Zivilisation. Bertelsmann, München.

Zanger, C. (2010): Stand und Perspektiven der Eventforschung - Eine Einführung. In: Zanger, C. (Hg.): Stand und Perspektiven der Eventforschung, Gabler, Wiesbaden, 1-12.

Zanger, C., Drengner, J. (2009): Eventmarketing. In: Bruhn, M., Esch, F.-R., Langner, T. (Hg.): Handbuch Kommunikation: Grundlagen - innovative Ansätze - praktische Umsetzungen, Gabler, Wiesbaden, 195-213.

Zanger, C., Sistenich, F. (1996): Eventmarketing. Bestandsaufnahme, Standortbestimmung und ausgewählte theoretische Ansätze zur Erklärung eines innovativen Kommunikationsinstruments. Marketing ZFP, 18. Jg., Heft 4, 233-242.

\section{Autoren}

Prof. Dr. rer. pol. Frank Sistenich

Fachgebiet Marketing

Prodekan

Leiter Institut für Marketing

Fachbereich Betriebswirtschaft/Wirtschaftsinformatik

Technische Hochschule Wildau [FH]

$\mathrm{T}+493375508-583$

frank.sistenich@th-wildau.de

Katrin Böckler

Technische Hochschule Wildau [FH] 
